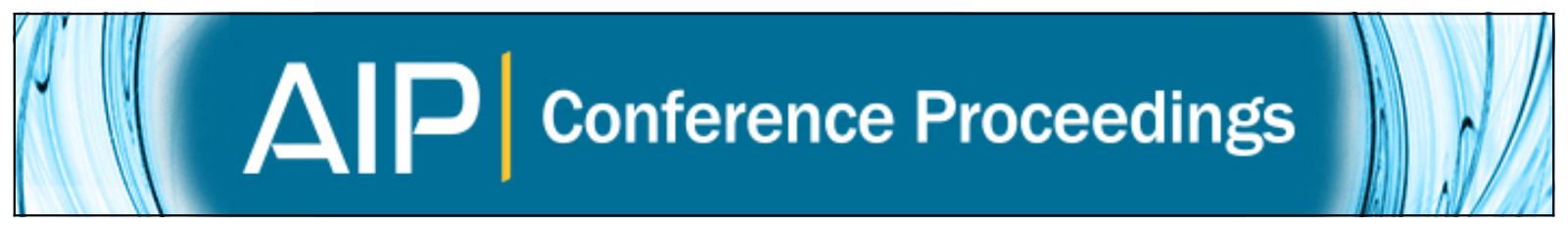

\title{
An Algorithm to Compute Abelian Subalgebras in Linear Algebras of Upper-Triangular Matrices
}

Manuel Ceballos, Juan Núñez, and Ángel F. Tenorio

Citation: AIP Conference Proceedings 1148, 53 (2009); doi: 10.1063/1.3225370

View online: http://dx.doi.org/10.1063/1.3225370

View Table of Contents: http://scitation.aip.org/content/aip/proceeding/aipcp/1148?ver=pdfcov

Published by the AIP Publishing

\section{Articles you may be interested in}

QHWM of the orthogonal type Lie subalgebra of the Lie algebra of matrix differential operators on the circle J. Math. Phys. 51, 093521 (2010); 10.1063/1.3483126

A subalgebra of Lie algebra A 2 and its associated two types of loop algebras, as well as Hamiltonian structures of integrable hierarchy

J. Math. Phys. 50, 053519 (2009); 10.1063/1.3122667

An efficient algorithm for computing the Baker-Campbell-Hausdorff series and some of its applications J. Math. Phys. 50, 033513 (2009); 10.1063/1.3078418

Classical Lie subalgebras of the Lie algebra of matrix differential operators on the circle J. Math. Phys. 42, 3735 (2001); 10.1063/1.1380252

On Lie algebras all nilpotent subalgebras of which are Abelian J. Math. Phys. 40, 4151 (1999); 10.1063/1.532955 


\title{
An Algorithm to Compute Abelian Subalgebras in Linear Algebras of Upper-Triangular Matrices
}

\author{
Manuel Ceballos*, Juan Núñez* and Ángel F. Tenorio \\ ${ }^{*}$ Departamento de Geometría y Topología. Facultad de Matemáticas. \\ Universidad de Sevilla. Aptdo. 1160. 41080-Seville (Spain). \\ ${ }^{\dagger}$ Departamento de Economía, Métodos Cuantitativos e H. Económica. Escuela Politécnica Superior. \\ Universidad Pablo de Olavide. Ctra. Utrera km. 1, 41013-Seville (Spain).
}

\begin{abstract}
This paper deals with the maximal abelian dimension of the Lie algebra $\mathrm{h}_{n}$, of $n \times n$ upper-triangular matrices. Regarding this, we obtain an algorithm which computes abelian subalgebras of $h_{n}$ as well as its implementation (and a computational study) by using the symbolic computation package MAPLE, where the order $n$ of the matrices in $h_{n}$ is the unique input needed. Let us note that the algorithm also allows us to obtain a maximal abelian subalgebra of $\mathrm{h}_{n}$.
\end{abstract}

Keywords: Maximal abelian dimension, solvable Lie algebra, algorithmic procedure, programming PACS: $02.20 . \mathrm{Sv} ; 02.10 . \mathrm{Hh} ; 02.10 . \mathrm{Ud}$

\section{INTRODUCTION}

The maximal abelian dimension of a given finite-dimensional Lie algebra g (i.e., the maximum among the dimensions of the abelian subalgebras of $g$ ) has been studied in previous papers. However, most of them (e.g. [5, 10]) consider abelian ideals instead of abelian subalgebras, being needed more restrictive hypotheses. As well as other papers like $[4,6]$, this does not assume such restrictions, but it considers all the subalgebras of the given Lie algebra g.

Jacobson [8] computed a classical bound for the dimension of any abelian subalgebra a of the matrix algebra $M_{n}(\mathbf{K})$, of $n \times n$ square matrices over a field $\mathbf{K}: \operatorname{dim}(\mathrm{a}) \leq\left[\frac{n^{2}}{4}\right]+1$, where $[x]$ denotes the integer part of $x$. Therefore the maximal abelian dimension $\mathscr{H}(\mathrm{g})$ of any given subalgebra $\mathrm{g}$ of $M_{n}(\mathbf{K})$ can be upper bounded by:

$$
\mathscr{H}(\mathrm{g}) \leq\left[\frac{n^{2}}{4}\right]+1= \begin{cases}k^{2}+1, & \text { if } n=2 k \\ k^{2}+k+1, & \text { if } n=2 k+1 .\end{cases}
$$

We have already studied the maximal abelian dimension of the Lie algebra $\mathrm{g}_{n}$, of $n \times n$ strictly upper-triangular matrices, by using an algorithmic method which computed abelian subalgebras in $[1,3]$. Besides, the law of $\mathrm{g}_{n}$ was computed by means of another algorithmic procedure in [2]. Now we are studying the maximal abelian dimension of the Lie algebra $\mathrm{h}_{n}$, of $n \times n$ upper-triangular matrices, by applying and adjusting the technics given in [3].

The Lie algebra $h_{n}$ is studied due to the following reasons: First, every finite-dimensional solvable Lie algebra is isomorphic to a subalgebra of some $h_{n}$ [11, Proposition 3.7.3]; and secondly, its applications to Physics are many and varied (e.g. [7, 9]).

\section{PRELIMINARIES}

Some preliminary concepts are recalled here, bearing in mind that the reader can consult [11] for a general overview on Lie algebras. This paper only considers finite-dimensional Lie algebras over the complex number field $\mathbf{C}$.

A Lie algebra $g$ is to be said solvable if its commutator central series becomes zero eventually:

$$
\mathscr{C}_{1}(\mathrm{~g})=\mathrm{g}, \mathscr{C}_{2}(\mathrm{~g})=[\mathrm{g}, \mathrm{g}], \ldots, \mathscr{C}_{k}(\mathrm{~g})=\left[\mathscr{C}_{k-1}(\mathrm{~g}), \mathscr{C}_{k-1}(\mathrm{~g})\right], \ldots, \mathscr{C}_{m}(\mathrm{~g}) \equiv\{0\}
$$

The maximal abelian dimension of a finite-dimensional Lie algebra $g$ is the maximum among the dimensions of its abelian subalgebras. This value will be denoted by $\mathscr{A}(\mathrm{g})$.

Given $n \in \mathbf{N}$, the complex solvable Lie algebra $\mathrm{h}_{n}$ is that whose vectors are the $n \times n$ upper-triangular matrices:

$$
h_{n}\left(x_{r, s}\right)=\left(\begin{array}{cccc}
x_{11} & x_{12} & \cdots & x_{1 n} \\
0 & x_{22} & \cdots & x_{2 n} \\
\vdots & \vdots & \ddots & \vdots \\
0 & \cdots & 0 & x_{n n}
\end{array}\right)
$$


where $x_{i j} \in \mathbf{C}$, for all $i, j \in \mathbf{N}$ with $1 \leq i \leq j \leq n$.

It is easy to prove that a basis of $h_{n}$ is formed by the vectors $X_{i j}=h_{n}\left(x_{r, s}\right)$ with $1 \leq i \leq j \leq n$ and such that:

$$
x_{r, s}= \begin{cases}1, & \text { if }(r, s)=(i, j), \\ 0, & \text { if }(r, s) \neq(i, j) .\end{cases}
$$

So the dimension of $h_{n}$ is $\frac{n(n+1)}{2}$. Besides, the nonzero brackets with respect to this basis are the following:

$$
\begin{array}{ll}
{\left[X_{i, j}, X_{j, k}\right]=X_{i, k},} & \forall i=1, \ldots, n-2, \quad \forall j=i+1, \ldots, n-1, \quad \forall k=j+1, \ldots, n ; \\
{\left[X_{i, i}, X_{i, j}\right]=X_{i, j}, \quad \forall i=1, \ldots, n-1, \quad \forall j=i+1, \ldots, n ;} \\
{\left[X_{k, i}, X_{i, i}\right]=X_{k, i}, \quad \forall k=1, \ldots, n-1, \quad \forall i=k+1, \ldots, n .}
\end{array}
$$

The center $Z\left(\mathrm{~h}_{n}\right)$ of $\mathrm{h}_{n}$ is generated by the vector $\sum_{i=1}^{n} X_{i, i}$. This vector has to belong to any abelian subalgebra which is not contained in another.

\section{ALGORITHM TO OBTAIN ABELIAN SUBALGEBRAS}

Next, we show an algorithmic procedure to obtain abelian subalgebras of the Lie algebra $h_{n}$. Before giving a general structure for this algorithm, we study the obtainment for $n<4$.

\section{Lie algebra $h_{n}$ with $n<4$}

Case $n=2$ : The Lie algebra $h_{2}$ is generated by the basis $\left\{X_{1,1}, X_{1,2}, X_{2,2}\right\}$ and the nonzero brackets with respect to this basis are $\left[X_{1,1}, X_{1,2}\right]=X_{1,2}$ and $\left[X_{1,2}, X_{2,2}\right]=X_{1,2}$. In this case, it is easy to prove that the 2-dimensional subalgebra $\left\langle X_{1,1}, X_{2,2}\right\rangle$ is abelian (i.e. the two vectors coming from the main diagonal). Consequently, $\mathscr{M}\left(\mathrm{h}_{2}\right)=2$.

Case $n=3:\left\{X_{1,1}, X_{1,2}, X_{1,3}, X_{2,2}, X_{2,3}, X_{3,3}\right\}$ is a basis of the Lie algebra $h_{3}$, whose nonzero brackets are the following:

$$
\left[X_{1,2}, X_{2,3}\right]=X_{1,3} ;\left[X_{1,1}, X_{1,2}\right]=X_{1,2} ;\left[X_{1,1}, X_{1,3}\right]=X_{1,3} ;\left[X_{2,2}, X_{2,3}\right]=X_{2,3} ;\left[X_{1,2}, X_{2,2}\right]=X_{1,2} ;\left[X_{1,3}, X_{3,3}\right]=X_{1,3} ;\left[X_{2,3}, X_{3,3}\right]=X_{2,3} \text {. }
$$

Step 1: Take the three vectors coming from the $3^{\text {rd }}$ column and remove the one coming from the $3^{\text {rd }}$ row. The abelian subalgebra $\left\langle X_{1,3}, X_{2,3}\right\rangle$ is obtained.

Step 2: Add the vectors coming from the $2^{\text {nd }}$ column and remove the ones coming from the $2^{\text {nd }}$ row (to avoid nonzero brackets). The dimension of the abelian subalgebra obtained here does not increase with respect to Step 1.

Step 3: Add the vector $X_{1,1}+X_{2,2}+X_{3,3}$, coming from the main diagonal and the only generator of $Z\left(\mathrm{~h}_{3}\right)$. Hence, the 3-dimensional abelian subalgebra $\left\langle X_{1,2}, X_{1,3}, X_{1,1}+X_{2,2}+X_{3,3}\right\rangle$ is obtained.

\section{A general structure for the algorithm}

Now we explain the algorithm to obtain abelian subalgebras. This depends on the parity of $n$, as it can be seen next. Hence, two possible cases have to be considered:

Case 1: $n$ is even and $n \geq 4$ (i.e., $n=2 k$, with $k \in \mathbf{N} \backslash\{1\}$ ). The general reasoning consists on considering the vectors in the basis of $\mathrm{h}_{n}$. When a vector coming from the $i^{\text {th }}$ column is chosen, only the vectors coming from the $i^{\text {th }}$ row lead to nonzero brackets. To avoid nonzero brackets, the vectors coming from the $i^{\text {th }}$ row have to be removed.

Step 1: $(2 k)^{\text {th }}$ column. Add the $2 k$ vectors coming from the $(2 k)^{\text {th }}$ column and remove the unique vector coming from the $(2 k)^{\text {th }}$ row. In this way, the abelian subalgebra $\left\langle X_{1,2 k}, \ldots, X_{2 k-1,2 k}\right\rangle$ is obtained.

Step $2 k-i+1: i^{\text {th }}$ column, with $2 k>i>k+1$. Add the $i$ vectors coming from the $i^{\text {th }}$ column to the generators of the previous step and remove the $2 k-(i-1)$ vectors coming from the $i^{\text {th }}$ row. In this way, we obtain an abelian subalgebra whose dimension increases $2 i-2 k-1$ with respect to the already obtained in the previous step. The dimension really increases if and only if $2 i-2 k-1>0$. Since this inequality is equivalent to $i>k+1 / 2, k$ is the last step in which the dimension of the abelian subalgebra increases.

Step $k$ : $(k+1)^{\text {th }}$ column. Add the $k+1$ vectors coming from the $(k+1)^{\text {th }}$ column and remove the $k$ ones coming from the $(k+1)^{\text {th }}$ row. 
The routine mas computes both the basis of a maximal abelian subalgebra of $\mathrm{h}_{n}$ and the dimension of this subalgebra (i.e. the maximal abelian dimension). Its unique input is the natural number $n$, corresponding to the order of the matrices in $\mathrm{h}_{n}$. We have defined the local variables $L, M, P, Q$ and $i$, where $L, M, P$ and $Q$ are sets and $i$ is a natural number. $L$ and $M$ store the vectors to be added and removed, respectively. $P$ stores the set difference $L \backslash M$. Finally, $Q$ has a unique element: the generator of $Z\left(h_{n}\right)$. Hence, the routine computes these sets in each step, returning the union $P \cup Q$ (i.e. a maximal abelian subalgebra) and its cardinal (i.e. the maximal abelian dimension).

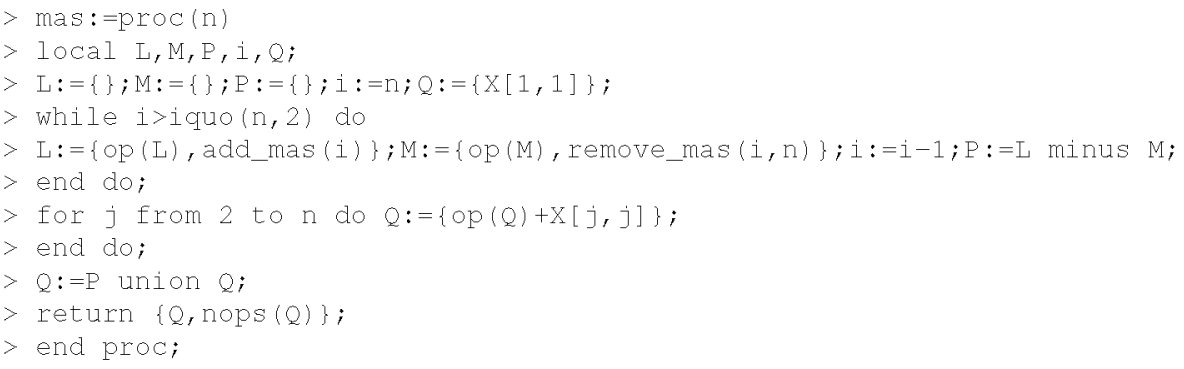

\section{COMPUTATIONAL STUDY}

The algorithm was implemented by using MAPLE 9.5 and was run in an Intel Core 2 Duo T 5600 with a $1.83 \mathrm{GHz}$ processor and 2.00 GB of RAM. Table 1 shows some computational data about this implementation for $n<2000$. Starting from $n=2$, the computational time is apparently double when the order $n$ is increased fifty units. In this way, for $n=1000$, the routine runs about 1 minute to compute a maximal abelian subalgebra of $h_{1000}$. For orders closer to 2000 , some problems arise in relation to an insufficient computational capacity. These problems are motivated by the need of a very high capacity of memory (almost $2 \mathrm{~GB}$ ). In any case, it does not suppose a serious problem because for this order the dimension of the Lie algebra would be about $2 \cdot 10^{6}$, which is not usually needed in practical situations.

TABLE 1. Computational time and used memory.

\begin{tabular}{|c|c|c|c||c|c|c|c|}
\hline $\begin{array}{c}\text { Input } \\
\text { order } n \text { ) }\end{array}$ & $\begin{array}{c}\text { Dimension } \\
\text { of } \mathrm{h}_{n}\end{array}$ & $\begin{array}{c}\text { Computational } \\
\text { time }\end{array}$ & $\begin{array}{c}\text { Used } \\
\text { memory }\end{array}$ & $\begin{array}{c}\text { Input } \\
\text { (order } \boldsymbol{n} \text { ) }\end{array}$ & $\begin{array}{c}\text { Dimension } \\
\text { of } \mathbf{h}_{n}\end{array}$ & $\begin{array}{c}\text { Computational } \\
\text { time }\end{array}$ & $\begin{array}{c}\text { Used } \\
\text { memory }\end{array}$ \\
\hline 2 & 3 & $0 \mathrm{~s}$ & $0 \mathrm{MB}$ & 400 & 80200 & $2.453 \mathrm{~s}$ & $18.75 \mathrm{MB}$ \\
\hline 50 & 1275 & $0 \mathrm{~s}$ & $0 \mathrm{MB}$ & 500 & 125250 & $5.468 \mathrm{~s}$ & $40.99 \mathrm{MB}$ \\
\hline 100 & 5050 & $0.046 \mathrm{~s}$ & $3.31 \mathrm{MB}$ & 600 & 180300 & $9.796 \mathrm{~s}$ & $63.55 \mathrm{MB}$ \\
\hline 150 & 11325 & $0.109 \mathrm{~s}$ & $5.69 \mathrm{MB}$ & 800 & 320400 & $27 \mathrm{~s}$ & $157.04 \mathrm{MB}$ \\
\hline 200 & 20100 & $0.266 \mathrm{~s}$ & $7.56 \mathrm{MB}$ & 1000 & 500500 & $59.266 \mathrm{~s}$ & $353.81 \mathrm{MB}$ \\
\hline 250 & 31375 & $0.562 \mathrm{~s}$ & $11.88 \mathrm{MB}$ & 1250 & 781875 & $156.641 \mathrm{~s}$ & $645.26 \mathrm{MB}$ \\
\hline 300 & 45150 & $0.969 \mathrm{~s}$ & $15.19 \mathrm{MB}$ & 1500 & 1125750 & $303.499 \mathrm{~s}$ & $1071.24 \mathrm{MB}$ \\
\hline 350 & 61425 & $1.609 \mathrm{~s}$ & $16.31 \mathrm{MB}$ & 1750 & 1532125 & $522.061 \mathrm{~s}$ & $1701.19 \mathrm{MB}$ \\
\hline
\end{tabular}

After carrying out a brief statistical study about the relation between the computational time and the memory used to compute the maximal abelian dimension of $h_{n}$, we can assert that the memory used depends on the computational time and viceversa. Indeed, this dependence is expressed by a very strong positive linear correlation. Besides, both the memory used and the computational time seem to be exponentially related to the order $n$.

\section{REFERENCES}

1. J. C. Benjumea, F. J. Echarte, J. Núñez and A. F. Tenorio, Extracta Math. 19, 269-277 (2004).

2. J. C. Benjumea, J. Núñez and A. F. Tenorio, "A computational method to obtain the law of the nilpotent Lie algebras $g_{n} "$, in Proceedings of Transgressive Computing 2006, edited by J. G. Dumas. Granada, 2006, pp. 53-62.

3. J. C. Benjumea, J. Núñez and A. F. Tenorio, Theor. Math. Phys. 152, 1225-1233 (2007).

4. R. Campoamor-Stursberg, Acta Phys. Polon. B 37, 2745-2760 (2006).

5. C. Caranti, S. Mattarei and F. Newman, Trans. Amer. Math. Soc. 349, 4021-4051 (1997).

6. D. Gorenstein, R. Lyons and R. Solomon, The classification of the finite simple groups, vol. 3, AMS, Providence, 1997.

7. S. Hervik, J. Geom. Phys. 52, 298-312 (2004).

8. N. Jacobson, Bull. Amer. Math. Soc. 50, 431-436 (1944).

9. P. J. Olver, Applications of Lie Groups to Differential Equations, Springer, New York, 1986.

10. J.-L. Thiffeault and P. J. Morrison, Phys. D 136, 205-244 (2000).

11. V. S. Varadarajan, Lie Groups, Lie Algebras and Their Representations, Springer, New York, 1984. 